\title{
Treatment outcomes of esophageal cancer in Eastern Africa: protocol of a multi-center, prospective, observational, open cohort study
}

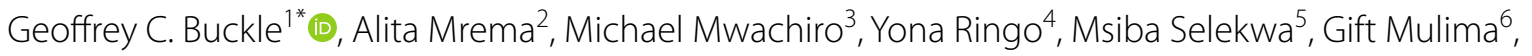
Fatma F. Some”, Blandina T. Mmbaga ${ }^{8,9}$, Gita N. Mody ${ }^{10}$, Li Zhang ${ }^{1}$, Alan Paciorek ${ }^{1}$, Larry Akoko ${ }^{5}$, Paul Ayuo ${ }^{7}$, Stephen Burgert ${ }^{3}$, Elizabeth Bukusi ${ }^{11}$, Anthony Charles ${ }^{10}$, Winnie Chepkemoi ${ }^{3}$, Gladys Chesumbai ${ }^{7}$, Bongani Kaimila ${ }^{12}$, Aida Kenseko ${ }^{13}$, Kitembo Salum Kibwana ${ }^{4}$, David Koech ${ }^{13}$, Caren Macharia ${ }^{3}$, Ezekiel N. Moirana ${ }^{14}$, Beatrice Paul Mushi ${ }^{5}$, Alex Mremi ${ }^{8}$, Julius Mwaiselage ${ }^{2}$, Ally Mwanga ${ }^{5}$, Jerry Ndumbalo ${ }^{2}$, Gissela Nvakunga ${ }^{8}$, Mamsau Ngoma ${ }^{2}$, Margaret Oduor $^{7}$, Mark Oloo $^{13}$, Jesse Opakas ${ }^{13}$, Robert Parker ${ }^{3,15}$, Saruni Seno ${ }^{13}$, Ande Salima ${ }^{12}$, Furaha Servent ${ }^{8}$, Andrew Wandera ${ }^{7}$, Kate D. Westmoreland ${ }^{10,12}$, Russell E. White ${ }^{3,15}$, Brittney Williams ${ }^{10}$, Elia J. Mmbaga ${ }^{5}$, Katherine Van Loon ${ }^{1}$ and of the African Esophageal Cancer Consortium (AfrECC)

\begin{abstract}
Background: Esophageal squamous cell carcinoma (ESCC) is a major cause of cancer morbidity and mortality in Eastern Africa. The majority of patients with ESCC in Eastern Africa present with advanced disease at the time of diagnosis. Several palliative interventions for ESCC are currently in use within the region, including chemotherapy, radiation therapy with and without chemotherapy, and esophageal stenting with self-expandable metallic stents; however, the comparative effectiveness of these interventions in a low resource setting has yet to be examined.
\end{abstract}

Methods: This prospective, observational, multi-center, open cohort study aims to describe the therapeutic landscape of ESCC in Eastern Africa and investigate the outcomes of different treatment strategies within the region. The 4.5-year study will recruit at a total of six sites in Kenya, Malawi and Tanzania (Ocean Road Cancer Institute and Muhimbili National Hospital in Dar es Salaam, Tanzania; Kilimanjaro Christian Medical Center in Moshi, Tanzania; Tenwek Hospital in Bomet, Kenya; Moi Teaching and Referral Hospital in Eldoret, Kenya; and Kamuzu Central Hospital in Lilongwe, Malawi). Treatment outcomes that will be evaluated include overall survival, quality of life (QOL) and safety. All patients ( $\geq 18$ years old) who present to participating sites with a histopathologically-confirmed or presumptive clinical diagnosis of ESCC based on endoscopy or barium swallow will be recruited to participate. Key clinical and treatment-related data including standardized QOL metrics will be collected at study enrollment, 1 month following treatment, 3 months following treatment, and thereafter at 3-month intervals until death. Vital status and QOL data will be collected through mobile phone outreach.

*Correspondence: geoffrey.buckle@ucsf.edu

${ }^{1}$ UCSF Helen Diller Family Comprehensive Cancer Center, University

of California, San Francisco, 505 Parnassus Ave, M1296, San Francsico, CA

94143, USA

Full list of author information is available at the end of the article

(c) The Author(s) 2022. Open Access This article is licensed under a Creative Commons Attribution 4.0 International License, which permits use, sharing, adaptation, distribution and reproduction in any medium or format, as long as you give appropriate credit to the original author(s) and the source, provide a link to the Creative Commons licence, and indicate if changes were made. The images or other third party material in this article are included in the article's Creative Commons licence, unless indicated otherwise in a credit line to the material. If material is not included in the article's Creative Commons licence and your intended use is not permitted by statutory regulation or exceeds the permitted use, you will need to obtain permission directly from the copyright holder. To view a copy of this licence, visit http://creativecommons.org/licenses/by/4.0/. The Creative Commons Public Domain Dedication waiver (http://creativeco mmons.org/publicdomain/zero/1.0/) applies to the data made available in this article, unless otherwise stated in a credit line to the data. 
Discussion: This study will be the first study to prospectively compare ESCC treatment strategies in Eastern Africa, and the first to investigate QOL benefits associated with different treatments in sub-Saharan Africa. Findings from this study will help define optimal management strategies for ESCC in Eastern Africa and other resource-limited settings and will serve as a benchmark for future research.

Trial registration: This study was retrospectively registered with the ClinicalTrials.gov database on December 15, 2021, NCT05177393.

Keywords: Esophageal squamous cell carcinoma, Esophageal cancer, Survival, Quality of life, Comparative effectiveness, Africa, Eastern Africa

\section{Background}

Esophageal cancer (EC) is one of the leading causes of cancer morbidity and mortality worldwide. Most recent estimates from GLOBOCAN report that EC is the eight most common cancer globally with 604,000 annual incident cases and the sixth leading cause of cancer-related mortality with 544,000 deaths each year [1]. Nearly $80 \%$ of all EC cases occur in low- and middle-income countries (LMICs), where esophageal squamous cell carcinoma (ESCC) is the dominant histology [2]. Incidence rates for ESCC exhibit substantial geographic variation globally with several regions impacted by a disproportionately high burden of this disease. Eastern Africa has been identified as one of these geographic "hot spots," along with north-eastern Iran, Central Asia, north-central China, and southern South America [2, 3]. In Eastern Africa, EC is the fifth leading cause of cancer mortality [1], with ESCC comprising 90\% of cases [4].

Limited research has been published on the treatment of ESCC in sub-Saharan Africa [5]. In this setting, more than $90 \%$ of patients present with advanced or inoperable disease, and few are candidates for treatment with curative intent [6-8]. Palliative treatment strategies for advanced ESCC thus dominate the therapeutic landscape and include stenting for malignant obstruction with selfexpandable metallic stents (SEMS), radiotherapy, chemoradiotherapy, and brachytherapy. Use of each of these treatment modalities is supported by data that originates from high-income countries (HICs) [9]. The unique challenges of delivering care in resource-constrained settings, however, limit the generalizability of these findings to many African settings. In a previous systematic review of all studies on EC treatment in Africa [5], we identified only four prospective case series evaluating treatment of advanced ESCC in Eastern Africa, three of which reported outcomes of palliative stenting at two sites in Kenya and Malawi [10-12] and one that reported outcomes of palliative radiation in Ethiopa [13]. To date, no study has prospectively compared treatment modalities for advanced ESCC in Eastern Africa. Furthermore, little is known regarding the impact of different ESCC treatments on patients' quality of life (QOL) in this setting. Of all the studies identified in our prior systematic review, only one examined QOL as an outcome metric [13].

With recent enhancement of cancer services in subSaharan Africa, the absence of context-specific data on treatment outcomes for ESCC is manifest. Expanded availability of chemotherapy, radiotherapy, and SEMS in Eastern Africa [14], has brought to light important questions regarding optimal management strategies for ESCC in this context. To address these questions, we developed a study protocol that aims to investigate the therapeutic landscape of ESCC in Eastern Africa and compare outcomes of different treatment approaches in use across the region. The Treatment Outcomes of Esophageal Cancer in Eastern Africa (TOEC-Eastern Africa) study is a prospective, observational, open cohort study of treatment strategies for ESCC that will recruit patients at six centers in Kenya, Malawi and Tanzania. This multi-center study has been established as a collaborative effort within the African Esophageal Cancer Consortium (AfrECC) [15].

\section{Methods}

Study objectives

The goals of the TOEC-Eastern Africa study are:

1) To describe the treatment patterns for ESCC at referral hospitals in Eastern Africa.

2) To investigate the outcomes of different ESCC treatments in Eastern Africa, specifically overall survival, QOL, and safety, with a particular focus on palliative interventions including chemotherapy, radiotherapy with or without chemotherapy, esophageal stenting with SEMS, and supportive care.

3) To examine process measures related to the delivery of ESCC treatment at referral hospitals in Eastern Africa, including time from presentation to initiation of treatment, total time to deliver an intervention, and total number of days hospitalized during and after ESCC treatment.

4) To assess utilization of healthcare resources by ESCC treatment approach, as measured by hospitalization events (total number of times hospitalized and 
cumulative days hospitalized) and need for subsequent intervention for recurrent or persistent dysphagia.

\section{Study design}

This study is designed as a multi-center, prospective, observational, open cohort study. Initiation of recruitment has been staggered across sites, with the earliest commencing in February 2019 and additional sites beginning recruitment in 2021. Enrollment is scheduled to complete in December 2022, with followup to complete in June 2023. In total, approximately 2476 patients are expected to be enrolled in the study. Patients will be followed to collect key clinical and treatment-related data including overall survival and QOL metrics until death or loss to follow up. QOL metrics will be assessed at the time of study enrollment, prior to the initiation of treatment, and during post-treatment follow up ( 1 month after initiation of treatment, 3 months after initiation of treatment, and then every 3 months until death or loss to follow up). The accrual period is expected to range between 22 and 47 months across sites. Duration of follow up will differ by site due to the staggered initiation of recruitment, however, study participants will be followed for at least 6 months and up to a total of 53 months.

\section{Study settings}

Participating sites are member institutions of AfrECC [15]. A total of six sites across Eastern Africa will participate, including: Ocean Road Cancer Institute (ORCI) and Muhimbili National Hospital (MNH) in Dar es Salaam, Tanzania; Kilimanjaro Christian Medical Center (KCMC) in Moshi, Tanzania; Tenwek Hospital in Bomet, Kenya; Moi Teaching and Referral Hospital (MTRH) in Eldoret, Kenya; and Kamuzu Central Hospital (KCH) in Lilongwe, Malawi (Fig. 1). Each site serves as a referral center for

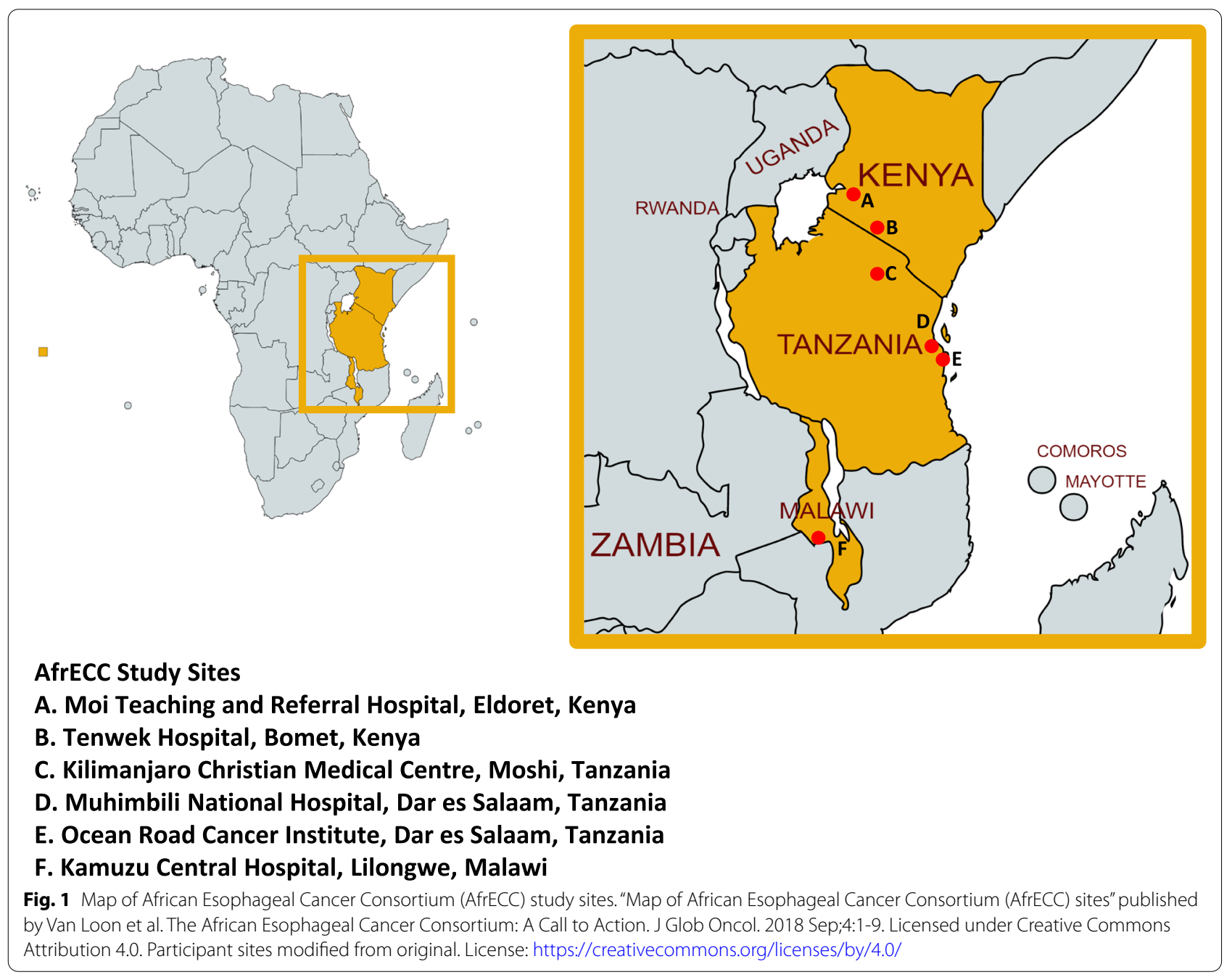


ESCC within the region. A summary of key characteristics and diagnostic and therapeutic services available at each study site is provided in Table 1.

In Tanzania, MNH is a public teaching hospital and national referral hospital affiliated with Muhimbili University of Health and Allied Sciences (MUHAS). MNH hosts 1500 beds, admitting 1000 to 1200 inpatients per week and providing care to more than 1000 outpatients per day. Following diagnosis, many of the cancer cases warranting chemotherapy and/or radiation therapy are referred to ORCI, the national cancer referral center in Tanzania, which hosts 273 beds. Muhimbili Academic Medical Center (MAMC) is a recently established public hospital affiliated with MNH. MAMC is located on the Mloganzila campus approximately $30 \mathrm{~km}$ from ORCI and $\mathrm{MNH}$. Endoscopy services are available at both $\mathrm{MNH}$ and MAMC. KCMC is a public teaching hospital and zonal referral hospital located in Moshi, Tanzania. KCMC hosts 630 inpatient beds and sees an estimated 500-800 outpatients per day. It serves as the teaching hospital for the Kilimanjaro Christian Medical University College and hosts the Kilimanjaro Clinical Research Institute (KCRI), an affiliated research institution.

In Kenya, Tenwek Hospital is a faith-based hospital and tertiary care center located in the southwestern Rift Valley Province in Bomet County. Tenwek Hospital hosts 361 inpatient beds and sees approximately 140,000 annual outpatient visits. MTRH is a 990-bed tertiary referral and teaching hospital in Eldoret, Kenya. The hospital serves residents of the Western Kenya Region, parts of eastern Uganda and South Sudan with a catchment area that covers 22 counties and extends $150 \mathrm{~km}$ to the Kenya-Uganda border, and a population of about 24 million people. MTRH has 1200 inpatients at any one time and provides care to 1500 outpatients per day. It has several specialist outpatient clinics under Chandaria Cancer and Chronic Disease Centre (CCCDC). Following diagnosis, oncology patients are referred to Oncology clinic for chemotherapy, radiotherapy or palliative/supportive care. Endoscopy services are available at MTRH and other private hospitals in Eldoret town. MTRH serves as a teaching hospital for Moi University College of Health Sciences and other medical training institutes.

In Malawi, $\mathrm{KCH}$ is an 800-bed tertiary referral hospital in Lilongwe. It serves six districts of the central region of Malawi with a population of about 6 million people. The Malawi Ministry of Health plans to open the first Malawi National Cancer Center in Lilongwe in late 2021 on the $\mathrm{KCH}$ campus. This will be the first dedicated cancer center offering multidisciplinary, comprehensive cancer care in the country, including radiotherapy, and will serve all of Malawi's $\sim 18$ million people. $\mathrm{KCH}$ has working relationships, both clinical and research, with the
College of Medicine under the University of Malawi and the Malawi College of Health Sciences.

\section{Outcome assessment Primary endpoint}

The primary endpoint for this study will be overall survival (OS), as defined by time elapsed from date of diagnosis to death or last follow up.

\section{Secondary endpoints}

Secondary endpoints that will be assessed include healthrelated QOL, as measured by the 'Modified Roseblatt Index', [16] major complications and treatment-related mortality. The 'Modified Rosenblatt Index' is a questionnaire assessing patient-reported outcomes previously developed for use among EC patients by Rosenblatt, et al., which has been adapted for use within a resourcelimited context (see Table 2) [16]. Parameters evaluated as part of this index are scored on a Likert scale assessing the presence and severity of the following: Dysphagia, Odynophagia, 'Chest and/or Back pain', World Health Organization (WHO) performance status, and 'Overall Well-being' since starting treatment. Secondary outcome measures related to dysphagia will also be assessed as dysphagia is commonly a dominant symptom among patients with advanced disease. These secondary measurements include time to achieve any dysphagia relief, time to achieve maximal dysphagia relief, duration of dysphagia relief, and dysphagia-adjusted survival.

\section{Exploratory endpoints}

Exploratory endpoints related to processes of care will also be measured, including time from presentation to initiation of ESCC treatment, time to deliver intervention (defined as total time elapsed from initiation of treatment to last day of treatment), and total number of days hospitalized during and after an intervention. Measures of healthcare utilization that will be assessed include hospitalization events (total number of times hospitalized and cumulative days hospitalized), and the proportion of patients receiving a subsequent intervention for recurrent or persistent dysphagia. Hospitalization is defined as admittance to the inpatient setting of a hospital (medical or surgical ward or intensive care unit) for a period of $\geq 24$ h per participant report.

\section{Eligibility criteria}

Patients aged $\geq 18$ years with a diagnosis of EC will be eligible for study inclusion. Expanded inclusion criteria will be used as not all patients with suspected EC undergo diagnostic biopsies due to the costs associated with biopsy and pathological review. Diagnostic criteria for EC will include either histopathological confirmation or 
Table 1 Characteristics of study sites in Eastern Africa

\begin{tabular}{|c|c|c|c|c|c|c|}
\hline Site & Tenwek Hospital & $\begin{array}{l}\text { Ocean Road } \\
\text { Cancer Institute } \\
\text { (ORCI) }\end{array}$ & $\begin{array}{l}\text { Muhimbili } \\
\text { National Hospital } \\
\text { (MNH) }\end{array}$ & $\begin{array}{l}\text { Kamuzu Central } \\
\text { Hospital (KCH) }\end{array}$ & $\begin{array}{l}\text { Moi Teaching and } \\
\text { Referral Hospital } \\
\text { (MTRH) }\end{array}$ & $\begin{array}{l}\text { Kilimanjaro } \\
\text { Christian Medical } \\
\text { Centre (KCMC) }\end{array}$ \\
\hline Location & Bomet, Kenya & $\begin{array}{l}\text { Dar es Salaam, } \\
\text { Tanzania }\end{array}$ & $\begin{array}{l}\text { Dar es Salaam, } \\
\text { Tanzania }\end{array}$ & Lilongwe, Malawi & Eldoret, Kenya & Moshi, Kilimanjaro \\
\hline Setting & Rural & Urban & $\begin{array}{l}\text { Urban (two cam- } \\
\text { puses, Upanga and } \\
\text { Mloganzila) }\end{array}$ & Urban & Urban & Urban \\
\hline Type of Hospital & Faith-based hospital & $\begin{array}{l}\text { National cancer } \\
\text { (specialized) referral } \\
\text { hospital }\end{array}$ & $\begin{array}{l}\text { National referral } \\
\text { hospital }\end{array}$ & $\begin{array}{l}\text { National referral } \\
\text { hospital }\end{array}$ & $\begin{array}{l}\text { National referral } \\
\text { hospital }\end{array}$ & $\begin{array}{l}\text { Zonal referral } \\
\text { hospital }\end{array}$ \\
\hline $\begin{array}{l}\text { Recruitment start } \\
\text { date }\end{array}$ & February 2019 & May 2019 & November 2019 & June 2021 & November 2021 & November 2021 \\
\hline $\begin{array}{l}\text { Recruitment end } \\
\text { date }^{\mathrm{a}}\end{array}$ & December 2022 & December 2022 & December 2022 & December 2022 & December 2022 & December 2022 \\
\hline $\begin{array}{l}\text { Estimated annual } \\
\text { new diagnoses } \\
\text { of EC }\end{array}$ & 250 & 150 & 167 & 300 & 325 & 90 \\
\hline $\begin{array}{l}\text { Forecasted annual } \\
\text { recruitment }\end{array}$ & 220 & 130 & 150 & 160 & 275 & 80 \\
\hline $\begin{array}{l}\text { Forecasted enroll- } \\
\text { ment (total) }\end{array}$ & 862 & 476 & 472 & 253 & 320 & 93 \\
\hline $\begin{array}{l}\text { Duration of enroll- } \\
\text { ment }\end{array}$ & 47 months & 44 months & 38 months & 19 months & 14 months & 14 months \\
\hline \multicolumn{7}{|c|}{ Diagnostic modalities available } \\
\hline Endoscopy & Yes & No & Yes & Yes & Yes & Yes \\
\hline Pathology & $\begin{array}{l}\text { Referred to outside } \\
\text { facility }\end{array}$ & Yes & Yes & Yes & Yes & Yes \\
\hline X-ray / ultrasound & Yes & Yes & Yes & Yes & Yes & Yes \\
\hline CT scan & Yes & Yes & Yes & Yes & Yes & Yes \\
\hline PET scan & No & No & No & No & No & No \\
\hline EUS & No & No & No & No & No & Mo \\
\hline \multicolumn{7}{|c|}{ Treatment modalities available } \\
\hline $\begin{array}{l}\text { Chemotherapy } \\
\text { Radiation }\end{array}$ & Yes & Yes & $Y_{e s}^{a}$ & Yes & Yes & Yes \\
\hline $\begin{array}{l}\text { Brachytherapy } \\
\text { (for EC) }\end{array}$ & No & No & No & No & No & No \\
\hline EBRT & No & Yes & No & Planned 2022 & Yes & Referred to ORCl \\
\hline $\begin{array}{l}\text { Esophageal } \\
\text { stents }\end{array}$ & Yes & No & Yes & Yes & Yes & Yes \\
\hline Esophagectomy & Yes & No & Yes & No & Yes & No \\
\hline \multicolumn{7}{|l|}{ Supportive care } \\
\hline Availability of TPN & Yes & Yes & Yes & Yes & Yes & Yes \\
\hline $\begin{array}{l}\text { Capacity for gas- } \\
\text { trostomy tubes }\end{array}$ & Yes & No & Yes & Yes & Yes & Yes \\
\hline $\begin{array}{l}\text { Intensive care } \\
\text { unit }\end{array}$ & Yes & No & Yes & Yes & Yes & Yes \\
\hline $\begin{array}{l}\text { Palliative care } \\
\text { team }\end{array}$ & Yes & Yes & No & Yes & Yes & Yes \\
\hline
\end{tabular}

Updated May 2021

CT Computed tomography, EC Esophageal cancer, EBRT External beam radiotherapy, EUS Endoscopic ultrasound, PET Positron emission tomography, TPN Total parental nutrition

${ }^{\text {a }}$ Chemotherapy is offered at $\mathrm{MNH}$; most EC patients, however, are referred to ORCI for chemotherapy 
clinical diagnosis based on barium swallow (esophagram) and/or upper endoscopy without biopsy. This clinical criteria has been shown to have a $>90 \%$ pre-test probability for the diagnosis of ESCC in Eastern Africa [17]. Access to a mobile phone is not a requirement for study entry; however, should a study participant lack access, this will be noted on baseline enrollment forms. Patients who have previously received treatment for EC will be eligible for study participation; in these cases, details of prior EC treatment will be abstracted from medical records, if available.

\section{Recruitment and consent of study participants}

Patients who present to participating AfrECC sites with symptoms concerning for or a new diagnosis of EC will be identified by the research team. Since many patients with ESCC are ill and subject to clinical deterioration, we will employ rapid case ascertainment strategies to identify all cases of EC seen for further care. Study teams are embedded in clinical care settings. At designated times each week, research coordinators will use the following strategies to identify eligible patients with suspected diagnoses of ESCC: 1 ) contact physicians caring for inpatients on both medical and surgical wards and review current ward rosters; 2) review the schedule of endoscopy cases to be performed, including diagnostic and therapeutic procedures (if applicable); 3) review pathology reports (if applicable); 4) contact clinicians seeing patients in the outpatient setting; and 5) review chemotherapy and radiation schedules to identify patients with plans to undergo ESCC treatment (if applicable).

Once potential study participants are identified, they will be approached by a research assistant to assess eligibility and willingness to participate in this study. Study participants will be thoroughly informed about all aspects of the study, including study follow up plans and all regulatory requirements for informed consent. Written informed consent will be obtained prior to study enrollment. The informed consent documents will be available in both English and the local language(s) at each site. Study participants will receive a phone card as a small token of compensation for participation, valued at 5 USD in Kenya and Tanzania and 10 USD in Malawi, in accordance with the local institutional standards.

\section{Data collection procedures Case report forms (CRFs)}

CRFs are comprised of both medical chart abstraction instruments and standardized questionnaires. CRFs collect socio-demographic details (including age, sex, ethnicity, location of permanent residence, highest level of education completed, self-reported annual household income, health insurance status), baseline clinical information, treatment-related data, as well as vital status and QOL metrics over long-term follow up (see Table 3).

\section{Patient interviews}

Interviews with study participants will be conducted at the following specified time-points: (i) at time of study enrollment); (ii) prior to the initiation of treatment (within 4 weeks); and (iii) during post-treatment follow up (1 month after initiation of treatment, 3 months after initiation of treatment, and then every 3 months until death or loss to follow up). Research assistants will collect data on sociodemographic characteristics, medical history, and baseline QOL through face-toface administration of oral questionnaires at the time of study enrollment. QOL assessments are based on the 'Modified Rosenblatt Index,' [16] a questionnaire comprised of six patient-reported outcomes assessing for the presence and severity of the following as scored on a Likert scale: Odynophagia, Dysphagia, 'Chest and/ or Back pain', WHO performance status, and 'Overall Well-being' since starting treatment (see Table 2). Subsequent interviews assessing vital status and QOL will be performed through mobile phone outreach. During post-treatment follow up, research assistants will attempt to contact patients a minimum of three times over the follow up interval before patients are classified as "lost to follow up." Outreach attempts will be made at varying times of the day on three different days of the week.

\section{Chart abstraction}

Clinical information will be abstracted from the patient's medical record at baseline (at time of study enrollment), during treatment(s), and following completion of any treatment(s). Clinical and treatment data collected through chart abstraction is summarized in Table 3. Treatment data will include dates of treatment, procedural details (stenting, esophagectomy, gastrostomy tube placement), radiation treatment regimens, chemotherapy regimens, major treatment- and disease-related complications, best response to treatment (if evaluated), reasons for discontinuing treatment or pursuing supportive care, and date and cause of death. Data will be collected on the time elapsed from diagnosis to initiation of treatment to account for any treatment delays that may explain differences in survival during analyses.

\section{Data management}

Data will be first collected using paper forms with subsequent entry of de-identified data into Research Electronic Data Capture (REDCap ${ }^{\mathrm{TM}}$ ), a secure web-based application for data storage. All study-related information, 


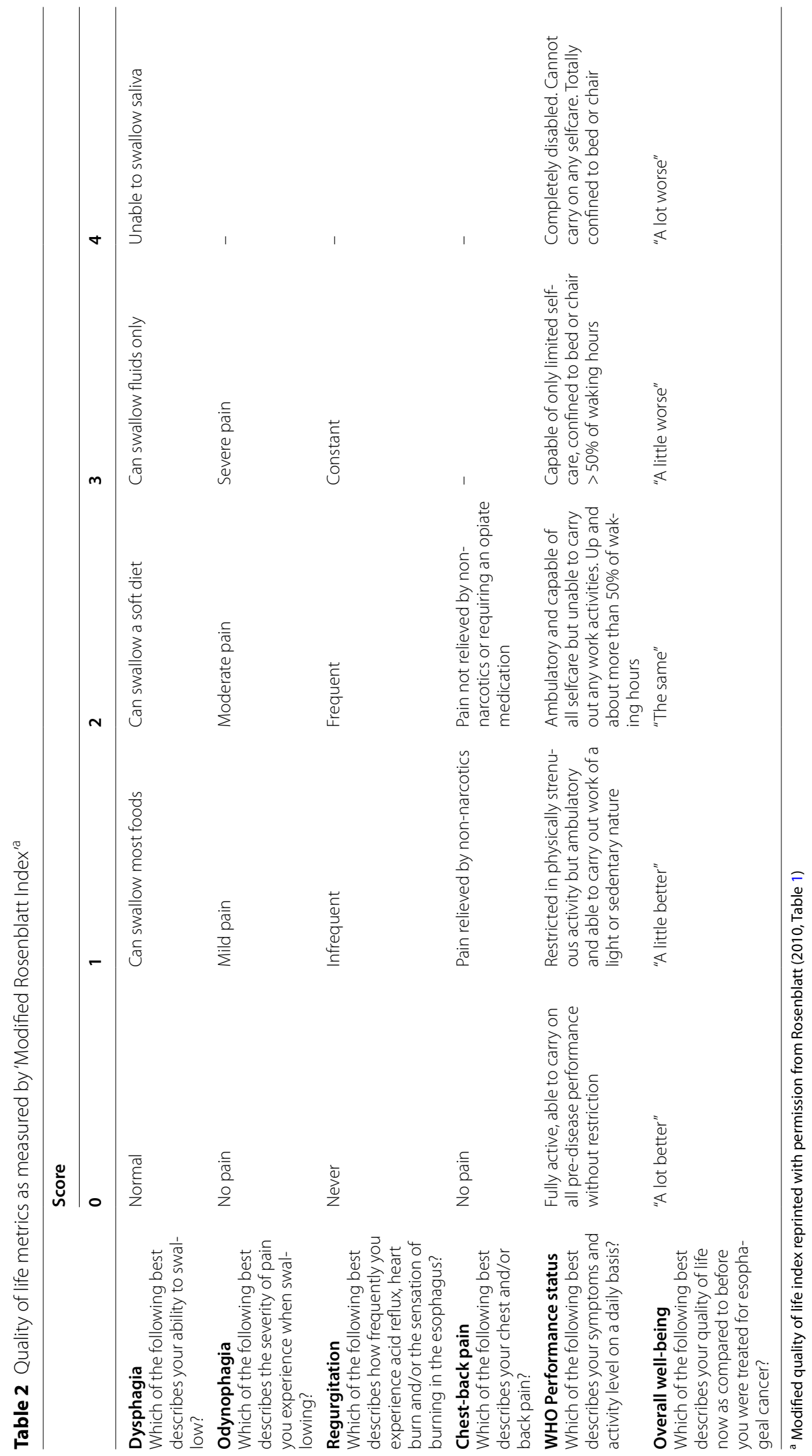


Table 3 Data collected during TOEC-Eastern Africa study

\begin{tabular}{|c|c|c|c|c|}
\hline Collected data & Baseline & Prior to treatment & During treatment & Follow up ${ }^{a}$ \\
\hline \multicolumn{5}{|l|}{ Sociodemographic } \\
\hline Age & $x$ & & & \\
\hline Sex & $\mathrm{x}$ & & & \\
\hline Ethnicity & $x$ & & & \\
\hline Occupation & $x$ & & & \\
\hline Location of permanent residence & $x$ & & & \\
\hline Highest level of education completed & $x$ & & & \\
\hline Annual household income & $x$ & & & \\
\hline Health insurance status & $x$ & & & \\
\hline Smoking and alcohol use & $x$ & & & \\
\hline \multicolumn{5}{|l|}{ Clinical data } \\
\hline Medical History & $x$ & & & \\
\hline HIV status (date of and last CD4 count, use of antiretrovirals) & $x$ & & & \\
\hline Date/method of diagnosis & $x$ & & & \\
\hline Histopathological data (if applicable) & $x$ & & & \\
\hline Endoscopic findings (location/length of tumor) & $x$ & & & \\
\hline Imaging (if applicable) ${ }^{b}$ & $x$ & & & \\
\hline Use of opiate medications for pain control & $x$ & $x$ & & $x$ \\
\hline Hospitalizations & & & $x$ & $x$ \\
\hline Vital status (including cause of death, if applicable) & & & $x$ & $x$ \\
\hline \multicolumn{5}{|l|}{ Treatment } \\
\hline Prior EC treatment (if applicable) & $x$ & & & \\
\hline $\begin{array}{l}\text { EC treatment received, including delays/interruptions in treatment } \\
\text { and reasons }\end{array}$ & & & $x$ & \\
\hline Complications & & & $x$ & \\
\hline Response assessment (if applicable) & & & $x$ & \\
\hline \multicolumn{5}{|l|}{ Quality of life } \\
\hline Modified Rosenblatt Index ${ }^{c}$ & $x$ & $x$ & & $x$ \\
\hline
\end{tabular}

EC Esophageal cancer

${ }^{\text {a }}$ Follow up assessments performed 1 month after initiation of treatment, 3 months after initiation of treatment, and then every 3 months until death or loss to follow up

${ }^{\mathrm{b}}$ Any imaging modality performed during baseline assessment, including chest x-ray, ultrasound, computed tomography, positron emissions tomography, etc.

'The Modified Rosenblatt Index assesses the following: Dysphagia, Odynophagia, 'Chest and/or Back pain,'WHO performance status, and 'Overall Well-being' since starting treatment

including completed paper forms for study participants, will be stored in locked cabinets in a secure location only accessible to authorized study personnel at each study site. Data for each participating site will be stored within a password-protected REDCap ${ }^{\text {TM }}$ data access group, with access limited to only authorized personnel from the recruiting AfrECC site. Electronic data capture forms within REDCap ${ }^{\mathrm{TM}}$ include data entry fields with programmed checks ('validation rules') for range and structure. University of California, San Francisco (UCSF) will serve as the data management coordinating center; however, each site will have access to its own data through this open-source platform.

\section{Sample size}

We aim to recruit all patients with ESCC based on histological confirmation or presumptive clinical diagnosis that meet the eligibility criteria at participating AfrECC sites. We forecast a total accrual of an estimated 2476 study participants across all six sites (see Table 1) over the duration of the study. This estimate is based on annual ESCC cases observed in recent case series at $\mathrm{KCH}$ (unpublished data), annual recruitment to a recent case-control study at KCMC [18], as well as annual accrual rates in the present study at ORCI, MNH, and Tenwek since study initiation (Tenwek Hospital, $n=220$ study participants per year; ORCI, $n=130$ per year; and $\mathrm{MNH}, n=150$ per year). Of the total study population, 
we estimate 1102 study participants will be treated with esophageal stenting, 145 with chemotherapy, 464 with radiotherapy, 212 with concurrent chemoradiation, 450 with supportive care alone, and 103 with esophagectomy. Based upon previous data that indicate median OS following stenting is 36 weeks [10], radiotherapy with or without chemotherapy is 27 weeks $[13,19]$, and chemotherapy is 14 weeks $[5,19]$, our primary analysis comparing OS of different palliative interventions for ESCC will have the following power to detect differences between each pairwise comparisons based upon alpha of 0.0167 (adjusting for 3 comparisons based on Bonferroni correction): stenting vs. radiotherapy with or without chemotherapy, power $=0.99$; stenting vs. chemotherapy, power $=1.00$; and radiotherapy with or without chemotherapy vs. chemotherapy, power $=1.00$.

\section{Statistical methods}

All patients consenting to study participation with a histopathologically-confirmed or clinical diagnosis of EC will be included in the analyses. Descriptive statistics will be used to describe the proportion of ESCC patients receiving treatment with each modality at participating AfrECC sites. We will assess the associations between demographic/clinicopathologic characteristics and the treatment modalities employed (chemotherapy, radiotherapy, chemoradiation, esophageal stenting with SEMS, esophagectomy, supportive care alone). Categorical variables and continuous variables will be compared among the groups using Pearson's Chi-squared test, and Analysis of Variance (ANOVA) (or Kruskal Wallis test when normality assumption is not held), respectively. Possible transformation (e.g., logarithm transformation) will be performed if the data is skewed.

OS will be defined in two ways: 1 ) from the date of initial diagnosis to the date of death or last patient contact; and 2) from date of initiation of treatment to date of death or the date of last patient contact. Kaplan-Meier method will be used to summarize OS and log-rank test will be used to compare survival curves among treatments overall and stratified by the study center. Furthermore, Cox proportional hazards (cph) modeling will be used to account for demographic and clinicopathological factors by including them as covariates. Proportionality of hazards for key covariates will be tested by examining the correlation between time and scaled Schoenfeld residuals and $\log$-log plots of survival. Important covariates that violate the proportionality assumption will be used to stratify the cph model. In many settings in Eastern Africa, long delays can occur between diagnosis and initiation of treatment. To mitigate immortal time bias that can emerge in time-to-event analyses of observational studies, two analytical approaches will be employed.
First, in analyses using OS defined by date of diagnosis, the time from diagnosis to the initial treatment will be added as a covariate. Secondly, in analyses of associations between different treatments and OS, treatment status will be included in Cox regression modeling as a timevarying covariate (defined as treated versus untreated). This approach allows for patients to accrue follow up time while awaiting treatment, while also accounting for any events that occur during the 'untreated' waiting period [20]. Our primary analysis will examine pairwise comparisons of: stenting vs. radiotherapy with or without chemotherapy, stenting vs. chemotherapy, and radiotherapy with or without chemotherapy vs. chemotherapy. Among patients receiving radiation, outcomes may differ between patients receiving radiotherapy alone as compared to concurrent chemoradiation. Exploratory analyses will examine these subgroups separately.

Cochran-Armitage test will be used to compare symptom metrics assessed using the 'Modified Rosenblatt Index' (Dysphagia, Odynophagia, 'Chest and/or Back pain', WHO performance status, and 'Overall Well-being' since starting treatment) between each of the different palliative EC treatments (chemotherapy, radiotherapy, chemoradiation, esophageal stenting, basic supportive care) at each time point: 1 month after initiation of treatment, 3 months after the initiation of treatment and then every 3 months thereafter. We will also test for withinsubject differences in scores between baseline (or pretreatment) and each of post-treatment time points by McNemar's Chi-squared test. In addition, generalized estimating equations (GEE) framework will be applied to analyze this longitudinal QOL data, where the treatment will be considered as the fixed effect and the subject-specific effects are the random effects.

Complications will be summarized as number of patients (\%) with 95\% confidence intervals and compared between treatment arms by Chi-squared test. Among palliative interventions, secondary outcome measures related to dysphagia will be compared using log-rank test and cph models to adjust covariates, including age, sex and baseline clinical covariates. 'Time to achieve any dysphagia relief' will be defined as time from treatment to improvement in dysphagia score of greater than or equal to 1. 'Duration of dysphagia relief' will be defined as time elapsed after treatment with any improvement in dysphagia from baseline. 'Dysphagia-adjusted survival' will be defined as the total time period with a dysphagia score of 0 to 2 .

Measures of healthcare utilization and process measures related to the delivery of ESCC treatment by treatment approach will be examined as exploratory endpoints. Time-to-event measures will be compared among treatment groups by log-rank test. Count of 
hospitalization events, and total number of days hospitalized during and after ESCC treatment will be compared among treatment groups by ANOVA (or Kruskal Wallis test when normality assumption is not held). Chisquared test will be used to compare the need for subsequent intervention for recurrent or persistent dysphagia by treatment approach. All analyses will be performed overall and within each site.

All statistical analyses will be performed using Stata or $\mathrm{R}$ statistical software. The significance level will be set for 0.05 for all analyses and multiple testing adjustments will be performed by Bonferroni correction.

\section{Reporting of study results}

The study results will be reported in adherence to Strengthening the Reporting of Observational Studies in Epidemiology (STROBE) criteria (see Supplemental Material for checklist) [21].

\section{Discussions}

The TOEC-Eastern Africa study will provide an overview of the therapeutic landscape of ESCC in Eastern Africa. The study will improve our understanding of the outcomes of different treatment strategies for ESCC currently in use throughout the region, including chemotherapy, radiotherapy with and without chemotherapy and esophageal stenting with SEMS. This study will address important an unmet need, as there is currently a paucity of data on the treatment of ESCC in low resource settings, particularly related to QOL [5]. The TOEC-Eastern Africa study will be the first within the region to prospectively compare treatment strategies for ESCC, and the first study in sub-Saharan Africa to evaluate QOL benefits associated with different treatments. Ultimately, findings from this study have potential to inform clinical practice guidelines for the management of ESCC in sub-Saharan Africa and other low resource settings.

Palliative interventions for EC have been an active area of research over the last several decades. Most of this research has taken place in HICs, with several RCTs having investigated best practices for palliation among patients with either ESCC or esophageal adenocarcinoma [9]. These studies highlight the tradeoffs between esophageal stenting with SEMS and the use of radiation-based therapies in patients with advanced disease $[22,23]$. SEMS have been shown to provide immediate dysphagia relief, but are prone to failure over time due to stent migration and/or recurrent obstruction. Radiation-based therapies, including external beam therapy (EBRT) with or without chemotherapy and brachytherapy, have delayed effects, but may offer more durable symptom control. Steyerberg et al. developed and validated a prognostic index to help guide treatment selection in this patient population, with the rationale that SEMS may be advantageous for those with short life expectancies [24]. Subsequent efforts to evaluate this index in a South African patient population found that it had poor predictive value in this context, highlighting the challenges of generalizing evidence from clinical trials in HICs to low resource settings [25]. Advanced dysphagia, severe weight loss, and malnutrition are all much more prevalent among patients with EC in these settings. Access to supportive care is often more limited as well. These differences, both in terms of patient characteristics and health system factors, underscore the importance of advancing research on treatment strategies for ESCC in sub-Saharan Africa, with the goal of optimizing treatment delivery in this context. Although RCTs remain the gold standard in comparative effectiveness research, several challenges emerge with this study design in Eastern Africa, including the ethics of randomizing patients who are often in poor general condition, and the time, infrastructure and resources needed for implementing well-designed clinical trials [26, 27].

The TOEC-Eastern Africa study is timely as efforts are currently underway to improve access to oncologic care throughout Eastern Africa as well as other regions in Africa. Historically, treatment approaches for ESCC in Eastern Africa have varied widely, with practice patterns determined by resource availability, as well as patients' access and financial means. Many cancer referral centers in the region have been able to offer patients with advanced ESCC esophageal stenting with SEMS, radiotherapy with or without chemotherapy, or chemotherapy alone. Few centers host the infrastructure and trained personnel to consider patients for all three treatment modalities. However, access to different treatment modalities is beginning to improve as governments invest in expanding the oncology workforce, developing infrastructure for radiotherapy, and essential medicines for cancer care become more broadly available. Parallel to these efforts, access to SEMS for esophageal stenting has improved in recent years as a result of coordinated efforts within AfrECC to launch the Stent Access Initiative [14]. This program, launched in collaboration with Boston Scientific and the Clinton Health Access Initiative, established the first ever sustainable supply chain of high-quality and affordable SEMS for AfrECC sites in Kenya, Tanzania, Malawi, and Zambia. This access program has been paired with a Training-of-Trainer program focused on building endoscopic skills for SEMS placement without fluoroscopy [28]. As treatment 
options for ESCC become more widely available for patients across the region, the need to better understand the risks and benefits of the treatments in use and establish evidence-based practice guidelines becomes all that more pressing.

In conclusion, Eastern Africa is impacted by an immense burden of ESCC. Despite being one of the most commonly diagnosed cancers in the region, there is a paucity of evidence to guide treatment decisions in the management of ESCC in this setting. The TOEC-Eastern Africa study leverages existing collaborations within AfrECC to collect robust data on ESCC treatment outcomes at six cancer referral centers in Kenya, Malawi, and Tanzania. Findings from this study have potential to improve our understanding of optimal treatment strategies for ESCC in Eastern Africa and other resource-limited settings. This coordinated prospective cohort study across six sites in three countries is novel and, if successful, may serve as a pragmatic approach to generate context-specific data regarding cancer therapies in subSaharan Africa.

\section{Study status}

The study first initiated recruitment at Tenwek Hospital in Kenya in February 2019, followed by Ocean Road Cancer Institute in Tanzania in May 2019. The multi-center study concept was presented by Dr. Geoffrey Buckle at the AfrECC Pre-Conference Meeting at the African Organization for Research and Training in Cancer (AORTIC) International Conference on Cancer in Africa in Maputo, Mozambique (November $5-8,2019)$. The study began recruitment at Muhimbili National Hospital in November 2019, Kamuzu Central Hospital in June 2021 and Moi Teaching and Referral Hospital in November 2021. Study recruitment is anticipated to complete December 2022 with follow up completing June 2023. The total duration of the study is estimated to be 53 months.

\footnotetext{
Abbreviations

AfrECC: African Esophageal Cancer Consortium; AORTIC: African Organization for Research and Training in Cancer; CCCDC: Chandaria Cancer and Chronic Disease Centre; CRF: Case reports forms; EBRT : External beam radiotherapy; EC: Esophageal cancer; ESCC: Esophageal squamous cell carcinoma; HDRILBT: High-dose-rate intraluminal brachytherapy; HICs: High-income countries; IRB: Institutional Review Board; IREC: Institutional Research and Ethics Committee; KCH: Kamuzu Central Hospital; KCMC: Kilimanjaro Christian Medical Centre; KCRI: Kilimanjaro Clinical Research Institute; KEMRI: Kenya Medical Research Institute; MAMC : Muhimbili Academic Medical Center; MNH: Muhimbili National Hospital; MTRH: Moi Teaching and Referral Hospital; MUHAS: Muhimbili University of Health and Allied Sciences; NACOSTI: National Commission for Science, Technology and Innovation; NHSRC: National Health Sciences Research Committee; ORCI: Ocean Road Cancer Institute; OS: Overall survival; QOL: Quality of life; RCT: Randomized Controlled Trial; REDCap ${ }^{\mathrm{TM}}$ : Research Electronic Data Capture; SEMS : Self-expandable metallic stent; TOEC: Treatment Outcomes of Esophageal Cancer; UCSF: University of California, San Francisco; UNC: University of North Carolina; WHO: World Health Organization.
}

\section{Supplementary Information}

The online version contains supplementary material available at https://doi. org/10.1186/s12885-021-09124-5.

Additional file 1. STROBE Checklist.

\section{Acknowledgments}

We are grateful for Emilie Kadhim's assistance with graphical design. We acknowledge the members of AfrECC for ongoing scientific collaboration in Eastern Africa.

\section{Authors' contributions}

All authors have contributed to the development of the study. GB, KVL and EM led the conception and design of the study in consultation with MS and $\mathrm{AM}$. $M M, G M, F S, B M, Y R, G M, L A, P A, S B, E B, A C, W C, G C, B K, A K, K K, D K, C M, E M, B M$, AM, JM, AM, JN, GN, MN, MO, MO, JO, RP, SS, AS, FS, AW, KW, RW and BW made contributions to the study design and adaptation to each study site. LZ and AP contributed to the design and statistical plan. GB drafted the manuscript with refinement by KVL. All authors contributed further revisions and have read and approved the final manuscript.

\section{Authors' information}

Not applicable.

\section{Funding}

This study received support from grants from the Celgene Cancer Care Links $^{\mathrm{TM}}$ and the University of California, San Francisco (UCSF) Resource Allocation Program following scientific peer review. Dr. Michael Mwachiro received funding from the GloCal Health Fellowship from the University of California Global Health Institute (D43TW009343). Dr. Geoffrey Buckle is supported by the Global Cancer Fellowship at UCSF Helen Diller Family Comprehensive Cancer Center and by the Maisin Foundation. Drs. Buckle and Van Loon receive research funding from the U.S. National Institutes of Health under Award Number UH3CA21 1457 and the U.S. National Cancer Institute under Award Number 3P30CA082103-21SA. Drs. Van Loon and Mmbaga receive funding from the Fogarty International Center (D43TW011958).

The funding agencies had no role in the design of this study and in the decision to publish, or in the preparation of the manuscript. Additionally, the funders will not have any role during the study conduct, including data collection, analyses, interpretation of the data or subsequent dissemination of results. The content is solely the responsibility of the authors and does represent the official views of the $\mathrm{NIH}, \mathrm{NCl}$, or Fogarty International Center.

\section{Availability of data and materials}

De-identified datasets generated from the current study are available upon reasonable request. Applications to access the datasets should be submitted to the corresponding author. All applications will be reviewed by the corresponding author and the TOEC study site leaders for approval.

\section{Declarations}

\section{Ethics approval and consent to participate}

The study protocol and site-specific informed consent forms, in English, Swahili, and Chichewa, were approved by the UCSF Institutional Review Board (IRB). The study protocol and informed consent documents were additionally approved by the MUHAS IRB, which serves as the IRB of record for MNH, ORCI and MAMC. The protocol was approved by the National Medical Research Institute in Tanzania. The protocol was additionally approved by the IRBs at Tenwek Hospital and the Kenya Medical Research Institute (KEMRI), which serve as the IRBs of record for Tenwek Hospital. The protocol was approved by the National Health Sciences Research Committee (NHSRC) in Malawi, the IRB of record for $\mathrm{KCH}$ and the University of North Carolina (UNC) IRB. The protocol was approved by the MTRH/Moi University Institutional Research and Ethics Committee (IREC) and the National Commission for Science, Technology and Innovation (NACOSTI) IRB for 
the addition of MTRH as a study site. The protocol was approved by the IRB of record for KCMC. Any modifications to the study protocol, informed consents or data collection instruments will require submission of a formal amendment and will be reviewed and approved by the responsible IRBs/ ethical committees at each site prior to implementation. Eligible study participants may only be included in this research study after providing written, informed consent.

\section{Consent for publication}

Not applicable.

\section{Competing interests}

The authors have no conflicts of interest to report

\section{Author details}

${ }^{1}$ UCSF Helen Diller Family Comprehensive Cancer Center, University of California, San Francisco, 505 Parnassus Ave, M1296, San Francsico, CA 94143, USA. ${ }^{2}$ Ocean Road Cancer Institute, Dar es Salaam, Tanzania. ${ }^{3}$ Tenwek Hospital, Bomet, Kenya. ${ }^{4}$ Muhimbili National Hospital, Dar es Salaam, Tanzania. ${ }^{5}$ Muhimbili University of Health and Allied Sciences, Dar es Salaam, Tanzania. ${ }^{6}$ Kamuzu Central Hospital, Lilongwe, Malawi. ${ }^{7}$ Moi University School of Medicine, Eldoret, Kenya. ${ }^{8}$ Kilimanjaro Clinical Research Institute, Kilimanjaro Christian Medical Centre, Moshi, Tanzania. ${ }^{9}$ Kilimanjaro Christian Medical University College, Moshi, Tanzania. ${ }^{10}$ University of North Carolina, Chapel Hill, USA. ${ }^{11}$ Kenya Medical Research Institute, Nairobi, Kenya. ${ }^{12}$ UNC-Project Malawi, Lilongwe, Malawi. ${ }^{13}$ Moi Teaching and Referral Hospital, Eldoret, Kenya. ${ }^{14}$ Kilimanjaro Christian Medical Centre, Moshi, Tanzania. ${ }^{15}$ Warren Alpert School of Medicine at Brown University, Providence, RI, USA.

Received: 12 May 2021 Accepted: 18 December 2021 Published online: 19 January 2022

\section{References}

1. Sung H, Ferlay J, Siegel RL, Laversanne M, Soerjomataram I, Jemal A, et al. Global cancer statistics 2020: GLOBOCAN estimates of incidence and mortality worldwide for 36 cancers in 185 countries. CA Cancer J Clin. 2021:caac.21660. https://doi.org/10.3322/caac.21660.

2. Malekzadeh R, Abnet CC, Dawsey SM. Oesophageal cancer: a tale of two malignancies. In: Wild CP, Weiderpass E, Stewart BW, editors. World cancer report: cancer research for cancer prevention: International Agency for Research on Cancer; 2020. http://publications.iarc.fr/586.

3. Cheng ML, Zhang L, Borok M, Chokunonga E, Dzamamala C, Korir A, et al. The incidence of oesophageal cancer in eastern Africa: identification of a new geographic hot spot? Cancer Epidemiol. 2015;39(2):143-9. https:// doi.org/10.1016/j.canep.2015.01.001.

4. Abnet CC, Arnold M, Wei W-Q. Epidemiology of esophageal squamous cell carcinoma. Gastroenterology. 2018;154(2):360-73. https://doi.org/10. 1053/j.gastro.2017.08.023.

5. Buckle GC, Mahapatra R, Mwachiro M, Akoko L, Mmbaga EJ, White RE, et al. Optimal management of esophageal cancer in Africa: a systemic review of treatment strategies. Int J Cancer. 2020. https://doi.org/10.1002/ IJC.33299.

6. Mannell A, Murray W. Oesophageal cancer in South Africa. A review of 1926 cases. Cancer. 1989;64(12):2604-8.

7. Come J, Castro C, Morais A, Cossa M, Modcoicar P, Tulsidâs S, et al. Clinical and pathologic profiles of esophageal cancer in Mozambique: a study of consecutive patients admitted to Maputo Central Hospital. J Glob Oncol. 2018:4:1-9. https://doi.org/10.1200/JGO.18.00147.

8. Mmbaga EJ, Deardorff KV, Mushi B, Mgisha W, Merritt M, Hiatt RA, et al. Characteristics of esophageal cancer cases in Tanzania. J Glob Oncol. 2018:4:1-10. https://doi.org/10.1200/JGO.2016.006619.

9. Dai Y, Li C, Xie Y, Liu X, Zhang J, Zhou J, et al. Interventions for dysphagia in oesophageal cancer (Review). 2014;(10). https://doi.org/10.1002/14651 858.CD005048.pub4.www.cochranelibrary.com.

10. White RE, Parker RK, Fitzwater JW, Kasepoi Z, Topazian M. Stents as sole therapy for oesophageal cancer: a prospective analysis of outcomes after placement. Lancet Oncol. 2009;10(3):240-6. https://doi.org/10.1016/ S1470-2045(09)70004-X.
11. Thumbs A, Borgstein E, Vigna L, Kingham TP, Kushner AL, Hellberg K, et al. Self-expanding metal stents (SEMS) for patients with advanced esophageal cancer in Malawi: an effective palliative treatment. J Surg Oncol. 2012;105(4):410-4. https://doi.org/10.1002/jso.23003.

12. Cotton RGH, Langer R, Leong T, Martinek J, Sewram V, Smithers M, et al. Coping with esophageal cancer approaches worldwide. Ann N Y Acad Sci. 2014;1325:138-58. https://doi.org/10.1111/nyas.12522.

13. Deressa BT, Tigeneh W, Bogale N, Buwenge M, Morganti AG, Farina E. Short-course 2-dimensional radiation therapy in the palliative treatment of esophageal Cancer in a developing country: a phase II study (Sharon project). Int J Radiat Oncol Biol Phys. 2019;106(1):67-72. https://doi.org/ 10.1016/j.jijobp.2019.10.004.

14. Mushi BP, Mwachiro MM, Buckle GC, Kaimila BN. Improving access to self-expanding metal stents for patients with esophageal cancer in eastern Africa: a stepwise implementation strategy original reports abstract. JCO Glob Oncol. 2021;7:118-26. https://doi.org/10.1200/ GO.20.00318.

15. Van Loon K, Mwachiro MM, Abnet CC, Akoko L, Assefa M, Burgert SL, et al. The African esophageal cancer consortium: a call to action. J Glob Oncol. 2018:2018(4):1-9. https://doi.org/10.1200/JG0.17.00163.

16. Rosenblatt E, Jones G, Sur RK, Donde B, Salvajoli JV, Ghosh-Laskar S, et al. Adding external beam to intra-luminal brachytherapy improves palliation in obstructive squamous cell oesophageal cancer: a prospective multi-centre randomized trial of the International Atomic Energy Agency. Radiother Oncol. 2010;97(3):488-94. https://doi.org/10.1016/j.radonc. 2010.09.001.

17. Van Loon K, Mmbaga E, Mushi B, Greer J, Benz S, Collisson E. Molecular determinants of esophageal cancer in Tanzania. In: 11th International Conference on Cancer in Africa: AORTIC. Kigali: African Organization for Research and Training in Cancer(AORTIC); 2017.

18. Mmbaga BT, Mwasamwaja A, Mushi G, Mremi A, Nyakunga G, Kiwelu I, et al. Missing and decayed teeth, oral hygiene and dental staining in relation to esophageal cancer risk: ESCCAPE case-control study in Kilimanjaro, Tanzania. Int J Cancer. 2020;148(10):2416-28. https://doi.org/ 10.1002/ijc.33433.

19. Dandara C, Robertson B, Dzobo K, Moodley L, Parker MI. Patient and tumour characteristics as prognostic markers for oesophageal cancer: a retrospective analysis of a cohort of patients at Groote Schuur Hospital. Eur J Cardio-Thoracic Surg Off J Eur Assoc CardioThoracic Surg. 2016;49(2):629-34. https://doi.org/10.1093/ejcts/ ezv135.

20. Karim ME, Gustafson P, Petkau J, Tremlett H, Shirani A, Zhao Y, et al. Comparison of statistical approaches for dealing with immortal time bias in drug effectiveness studies. Am J Epidemiol. 2016;184(4):325-35. https:// doi.org/10.1093/aje/kwv445.

21. von Elm E, Altman DG, Egger M, Pocock SJ, Gøtzsche PC, Vandenbroucke JP. The strengthening the reporting of observational studies in epidemiology (STROBE) statement: guidelines for reporting observational studies. Lancet. 2007;370(9596):1453-7. https://doi.org/10.1016/S0140-6736(07) 61602-X.

22. Homs MY, Essink-Bot M-L, Borsboom GJJ, Steyerberg EW, Siersema PD. Quality of life after palliative treatment for oesophageal carcinoma - a prospective comparison between stent placement and single dose brachytherapy. Eur J Cancer. 2004;40(12):1862-71. https://doi.org/10. 1016/j.ejca.2004.04.021.

23. Turrisi A, Hawes R, Palesch Y, Redmond C, Williams T, Reed C, et al. The SORTIE trial: palliation with stent (S) or radiation therapy (RT) 20 Gy in 5 fractions intervention for esophageal cancer dysphagia: a multicenter trial for T-4, M+/- squamous or adenocarcinoma of the esophagus. A randomized trial relief from dysphagia and quality of life (QOL) analysis. Int J Radiat Oncol. 2002;54(2):132. https://doi.org/10.1016/s0360-3016(02) 03284-4.

24. Steyerberg EW, Homs MYV, Stokvis A, Essink-Bot M-L, Siersema PD. Stent placement or brachytherapy for palliation of dysphagia from esophageal cancer: a prognostic model to guide treatment selection. Gastrointest Endosc. 2005;62(3):333-40. https://doi.org/10.1016/S0016-5107(05) 01587-7.

25. Loots E, Anderson F, Clarke DL, Mulder CJMT. Self-expandable metal stents in esophageal cancer in a high HIV prevalence area: a survival analysis and evaluation of prediction scores. Surg Laparosc Endosc Percu$\tan$ Tech. 2016;26(6):455-8. 
26. Ezeani A, Odedina F, Rivers D, Fatiregun O, Akinremi T. SWOT analysis of oncology clinical trials in Africa: a town hall report from the global congress on oncology clinical trials in blacks. J Glob Oncol. 2019;2019(5). https://doi.org/10.1200/JGO.19.00199.

27. Gopal S. Cancer trials in sub-Saharan Africa: aligning research and care summary points. 2017. https://doi.org/10.1371/journal.pmed.1002351.

28. Mwachiro MM, Mushi B, Buckle G, Kayamba V, Kamila B, Sedlack R, et al. A stepwise approach to implementation of esophageal stenting for palliation of esophageal cancer in East Africa. Gastrointest Endosc. 2020;91(6):AB533.

\section{Publisher's Note}

Springer Nature remains neutral with regard to jurisdictional claims in published maps and institutional affiliations.

- fast, convenient online submission

- thorough peer review by experienced researchers in your field

- rapid publication on acceptance

- support for research data, including large and complex data types

- gold Open Access which fosters wider collaboration and increased citations

- maximum visibility for your research: over $100 \mathrm{M}$ website views per year

At BMC, research is always in progress.

Learn more biomedcentral.com/submissions 